\title{
Sine oculis homeobox homolog 1 promotes DNA replication and cell proliferation in cervical cancer
}

\author{
DAN LIU $^{1 *}$, XIAO-XUE ZHANG ${ }^{1 *}$, BI-XIN XI ${ }^{1}$, DONG-YI WAN ${ }^{1}$, LI LI $^{1}$, JIN ZHOU $^{1}$, \\ WEI WANG ${ }^{2}$, DING MA ${ }^{1}$, HUI WANG ${ }^{1}$ and QING-LEI GAO ${ }^{1}$ \\ ${ }^{1}$ Cancer Biology Research Center, Tongji Hospital, Tongji Medical College, Huazhong University of \\ Science and Technology, Wuhan; ${ }^{2}$ Department of Obstetrics and Gynecology, Nanfang Hospital, \\ Southern Medical University Guangzhou, Guangzhou, P.R. China
}

Received March 19, 2014; Accepted May 26, 2014

DOI: $10.3892 / \mathrm{ijo} .2014 .2510$

\begin{abstract}
Malignant proliferation is the fundamental trait of tumor cells. The initiation of DNA replication represents a key process for cell proliferation, and has a marked impact on tumorigenesis and progression. Here we report that Sine oculis homeobox homolog 1 (SIX1) functions as a master regulator in DNA replication of cervical cancer cells. The expression of SIX1 was induced by the E7 oncoprotein of human papillomaviruses in cervical intraepithelial neoplasia and cervical cancer. The increase of SIX1 expression resulted in the upregulation of multiple genes related to the initiation of DNA replication, including the genes coding for the proteins in minichromosome maintenance complex (MCM2, MCM3, MCM6), DNA polymerase $\alpha$-primase complex (POLA1, PRIM1, PRIM2), clamp loader (RFC3, RFC4, RFC5), DNA polymerase $\delta$ complex (POLD3) and DNA polymerase $\varepsilon$ complex (POLE2). In line with this, the increase of SIX1 expression enhanced DNA synthesis, accelerated $\mathrm{G} 1$ to $\mathrm{S}$ phase progression, and promoted the proliferation of cervical cancer cells and the growth of cervical cancer. Consistently, knockdown of SIX1 could hamper DNA synthesis, slow down G1 to $\mathrm{S}$ phase progression, and suppress tumor cell proliferation and tumor growth. Importantly, SIX1 could more efficiently promote anchorage-independent cell growth. These results suggest that the increase of SIX1 expression could promote tumorigenesis, progression and invasive growth of cervical cancer by promoting DNA replication, and that targeting SIX1 may have significant therapeutic value in cervical cancer treatment.
\end{abstract}

Correspondence to: Dr Qing-Lei Gao, Cancer Biology Research Center, Tongji Hospital, Tongji Medical College, Huazhong University of Science and Technology, 1095 Jiefang Anv, Wuhan, P.R. China E-mail: qingleigao@hotmail.com

\section{${ }^{*}$ Contributed equally}

Key words: Sine oculis homeobox homolog 1, DNA replication, cell proliferation, cervical cancer

\section{Introduction}

Cervical cancer is ranked as the second cause of cancer deaths in women worldwide (1-3). Human papillomaviruses (HPV) are considered as the causative agents of most cervical cancers (1-3). The binding of HPV E6 and E7 oncoproteins to tumor-suppressor genes $p 53$ and $R B$ could result in impaired tumor-suppressor-gene function and eventual cell immortalization (2). Although recent studies have identified other equally important molecular events for malignant transformation of cervical epithelial cells, including the activation of certain tumor-promoting genes and pathways (1), E6 and E7 oncoproteins are widely recognized as the driving force in carcinogenesis of cervical epithelium. However, it is not clear whether and how E6 and E7 could promote the progression of cervical cancer after the tumor formation.

The immortalized cells resulting from transfection with HPV DNA have been found to express significantly higher levels of Sine oculis homeobox homolog 1 (SIX1), suggesting that HPV might be able to promote tumor cell proliferation by increasing SIX1 expression (4). SIX1 gene is one of homeobox genes which code for transcription factors functioning as master regulators in proliferation, apoptosis, migration and invasion (5). Similar to many cell cycle proteins, the expression of SIX1 is regulated in a cell cycle-specific manner, where it is transcriptionally upregulated at the G1/S boundary, highly expressed in $\mathrm{S}$ and $\mathrm{G} 2 / \mathrm{M}$ phases, then degraded via the ubiquitin-proteasome pathway (6). SIX1 is necessary in early embryogenesis, but its expression is lost in most well differentiated tissues. However, SIX1 has been found to be overexpressed in a variety of malignancies, showing the ability to promote tumor growth and metastasis (7). Moreover, SIX1 was reported to abolish the G2 cell cycle checkpoint and upregulate cyclin A1 to promote cell proliferation in breast cancer $(8,9)$. These results implied that SIX1 might play an important role in the proliferation of tumor cells and the development of tumor.

The ability of tumor cells to sustain the uncontrolled proliferation might be influenced by the factors that could modulate DNA replication, cell cycle, apoptosis-resistance, metabolic capacity, etc. In this study we investigated the mechanism underlying the effect of SIX1 on the proliferation of cervical 
cancer cells. The resultant data showed that SIX1 functioned as a master regulator in DNA replication. SIX1 upregulated multiple genes affecting the initiation of DNA replication, accelerated G1 to S phase progression, and promoted tumor cell proliferation. These results implied that SIX1 might play a crucial role in tumorigenesis and progression of cervical cancer, and also suggested that SIX1 might be a therapeutic target in cervical cancer treatment.

\section{Materials and methods}

Cells and transfection. Human cervical cancer cell lines C33a (HPV-negative), Siha and Caski (HPV-positive) were purchased from the American Type Culture Collection (Manassas, VA). Cells were transduced with CMV-Fluc-IRES-RFP lentiviral particles (GeneChem, Shanghai, China). The cells with stable transfection $\left(\mathrm{RFP}^{+}\right)$were isolated by FACS. RFP/luciferase-expressing cells were used in living imaging. In cell cycle analysis by flow cytometry, RFP-free cells were used.

The expression vectors for E6 and E7 were constructed as previously described (10). Briefly, the cDNAs coding for E6 and E7 were amplified from total RNA isolated from Caski cell lines using following primers: E6, 5'-GCGGCCGCC ACCATGTTTCAGGACCACAG-3' (sense) and 5'-CTGCGG CCGCGATTACAGCTGGGTTTTCTCT-3' (antisense); E7, 5'-GCGGCCGCCACCATGGCATGGCATGGAGATAC ACCT-3' (sense) and 5'-AGGCGGCCGCGATTATGGTTT CTGAGAACA-3' (antisense). The cDNAs were inserted into expression vector pcDNA3.1. C33a cells were transfected with plasmid pcDNA3.1-SIX1 (a kind gift from Kong-Ming Wu, Thomas Jefferson University, Philadelphia, PA), pcDNA3.1-E6, pcDNA3.1-E7 or pcDNA3.1 using Lipofectamine 2000 (Invitrogen, Life Technologies). ShSIX1(1) and shSIX1(2) shRNA lentiviral particles (GeneChem) were used to knockdown SIX1 expression, targeting 5'-CCAGCTCAGAAGAGG AATT-3' and 5'-CACGCCAGGAGCTCAAACT-3', respectively. Shcon, the shRNA not targeting any known gene, was used as control. Caski cells were transduced with SIX1 shRNA lentiviral particles, or control shRNA lentiviral particles. The cells with stable transfection were selected with G418 (C33a) and puromycin (Caski). The transfected cells were designated as C33a-3.1, C33a-E6, C33a-E7, C33a-SIX1, Caski-shcon, Caski-shSIX1(1) and Caski-shSIX1(2), respectively.

Immunohistochemistry. A tissue microarray (TMA) (CIN481) was purchased from Alenabio (Xi-an, China), containing cervical intraepithelial neoplasia (CIN) samples. Tumor samples were acquired by surgery from cancer patients without preoperative treatment, which was approved by the Ethics Committee of the Medical Faculty of Tongji Medical College. Informed consent was obtained from all subjects. The tumor samples were processed into TMA at Outdo Biotech Company (Shanghai, China). TMAs and tissue sections were subjected to immunohistochemical analysis as described previously (11), using the Avidin-Biotin Complex (ABC) Vectastain Kit (Zsgb-Bio, Beijing, China). Rabbit anti-human SIX1 antibody (Sigma-Aldrich) and rabbit antihuman Ki67 antibody (Santa Cruz Biotechnology) were used as primary antibodies. For semi-quantitative evaluation of SIX1 expression in tissue, staining intensity was graded
(0, absence; 1 , weak; 2 , moderate; 3 , strong), and assessed using an immunoreactivity-scoring system, HSCORE (12). The HSCORE was calculated using the following formula: HSCORE $=\sum \mathrm{Pi}(\mathrm{i}+1)$, where $\mathrm{i}$ is the staining intensity of tumor cells and $\mathrm{Pi}$ is the percentage of tumor cells at each level of intensity. The percentage of Ki67-positive tumor cells was measured as mean of five hot-spots on one slide from each tumor. Hot-spots were defined as areas with the highest Ki67 intensity. The slides were read by two pathologists and each data point represents the mean of the scores from two pathologists.

Assay of gene expression by real-time RT-PCR. Total RNA was extracted from cells with TRIzol reagent (Invitrogen). The relative quantity of mRNA was determined by real-time RT-PCR. Gapdh and EEFIAl were chosen as reference genes, which were reported as the most reliable combination in cervical cancer (13). The primer sequences are shown in Table I. The expression of genes was quantified using the comparative $\mathrm{C}_{\mathrm{T}}$ method. The expression level of each mRNA was normalized to that of GAPDH and EEFIAI mRNAs, and expressed as $\mathrm{n}$-fold difference relative to the control.

Western blot assay. Western blot assay was performed as described previously (14). The antibodies were purchased from Sigma-Aldrich, Biorbyt and Santa Cruz Biotechnology.

Bioinformatic analysis. The normalized RNA-sequencing data of 116 cases of cervical cancer samples was publicly available from TCGA database (https://tcga-data.nci.nih.gov/ tcga/tcgaHome2.jsp). Differential gene expression based on SIX1 expression category was determined using Bioconductor edgeR package (15) as described previously (16). Then, pathway analysis of differentially expressed genes was performed using Onto-Express from Onto-Tools package (17).

To further analyze the effect of SIX1 expression on DNA replication and cell cycle pathways (gene sets), Gene set enrichment analysis (GSEA) package was used as described previously (18). For this analysis, SIX1 expression was set as continuous label. Heat map was generated by GSEA.

BrdU incorporation. The BrdU colorimetric ELISA kit (Roche) was used to detect 5'-bromo-2'-deoxyuridine (BrdU) incorporated into newly synthesized DNA, according to the manufacturer's protocol. Briefly, tumor cells were incubated with BrdU solution for $2-16 \mathrm{~h}$ at $37^{\circ} \mathrm{C}$, then fixed and denatured and incubated with a peroxidase-conjugated antibody against BrdU for $1 \mathrm{~h}$ at room temperature. The cells were then incubated with the tetramethyl-benzidine (TMB) substrate solution and absorbance was read at $450 \mathrm{~nm}$ using a multiskan spectrum microplate reader ( $\mu$ Quant Bio-Tek Instruments, USA).

Flow cytometry analysis. Cells were harvested by trypsinization and fixed in $70 \%$ ice-cold ethanol overnight at $-20^{\circ} \mathrm{C}$, and then washed in PBS and incubated in RNase A (10 U/ml) and propidium iodide $(20 \mathrm{mg} / \mathrm{ml})$ for $30 \mathrm{~min}$ at room temperature. The cells in different phases were detected by flow cytometry on a FACS Calibur system (Becton-Dickinson) and analyzed using FlowJo software. Serum starvation was performed as described previously (19). 
Table I. Primers for real-time RT-PCR.

\begin{tabular}{lll}
\hline Gene & \multicolumn{1}{c}{ Sense primers } & \multicolumn{1}{c}{ Antisense primers } \\
\hline GAPDH & GACAGTCAGCCGCATCTTCT & TTAAAAGCAGCCCTGGTGAC \\
EEF1A1 & TGCGGTGGGTGTCATCAAA & AAGAGTGGGGTGGCAGGTATTG \\
MCM2 & TTGGCGTGAGTTGCGTATTC & GAGACTGAAAACGATTACAAACATC \\
MCM3 & CCACAGATGATCCCAACTTT & GTCCCATGTAGAAGGTTGTC \\
MCM4 & TGAACCTCTATACATGCAACGAC & CAGGGTAACGGTCAAAGAAGATT \\
MCM5 & TCACCAAGCAGAAATACCC & GTCCATGAGTCCAGTGAG \\
MCM6 & AGTGTGTGAGTCGATGAATA & CATTAAAGAGGAGCGAGCTT \\
MCM7 & GGAAATATCCCTCGTAGTATCAC & CTGAGAGTAAACCCTGTACC \\
POLA1 & AAAGATCCATTGGAGCTTCACC & TCAGCACGTTTAAGAGGAACAG \\
POLA2 & AGGAGCTAGAGACATTGTTTCCA & CTCGCTTCTGAGAACCCTTG \\
PRIM1 & ACATTCGCTACCAATCCTTCAAC & AGCTCCCAGCTTCACTGTATT \\
PRIM2 & TCTTCGAGAACAGGAGATTGTTG & CAGAGCATCAGCAAAAGGGAT \\
RFC1 & TTGTCATGGGTCGTGATAGTGG & CCTGGCATAGTCCGAATCAGAT \\
RFC2 & GTGAGCAGGCTAGAGGTCTTT & TGAGTTCCAACATGGCATCTTTG \\
RFC3 & GTGGACAAGTATCGGCCCTG & TGATGGTCCGTACACTAACAGAT \\
RFC4 & CCGCTGACCAAGGATCGAG & AGGGAACGGGTTTGGCTTTC \\
RFC5 & GACATGCGTAGGGCTCTGAAC & GTGCAGGTGTAGACAGTCTCC \\
POLD1 & ATCCAGAACTTCGACCTTCCG & ACGGCATTGAGCGTGTAGG \\
POLD2 & ATGTTTCTGAGCAGGCTGCC & TGGGGGAGCAGGTTGTGCTC \\
POLD3 & AAGCCATGCTAAAGGACAGTG & CATTGGTGGTCAGCTCATTGTT \\
POLD4 & ATCACTGATTCCTACCCGGTT & AGAGATGCCAGAGACTGCACT \\
POLE2 & TTGCGACCAGAAAGGGTTGT & TGATTTGGCAAGTCCAGATCCT \\
POLE3 & TGAGAAGCAACCCTTGTCATC & TCATCAACAGACTGACTGCATTC \\
POLE4 & GGCCCGAGGACCTAAACCT & ATGTGGCGTACAGCACGAAG \\
\hline & AAGGTCTTCCACTGTTTCTGTCTG & CCTTTATTCTGGTCCTCATCATTC \\
\hline
\end{tabular}

Tumor cell proliferation assay. Tumor cells were seeded at $2 \times 10^{3}$ per well into 96 -well plates. Using cell counting kit- 8 (CCK8, Boster, China), the proliferation of the cells at each time point was measured with multiskan spectrum microplate reader ( $\mu$ Quant Bio-Tek Instruments, USA) at $450 \mathrm{~nm}$ wavelength. Cell proliferation index was expressed as fold change related to day 0 .

Soft agar assay. Cells were suspended in $0.35 \%$ agar in DMEM ( $20 \%$ FBS) and plated $\left(1.5 \times 10^{3}\right.$ per well) on a layer of $0.5 \%$ agar in culture medium in 6-well plates. After two-week culture, colonies were counted and photographed by phase-contrast microscopy (Nikon, Japan). The size of 20 randomly chosen colonies per well was measured. The average size of colony was calculated as (length + width) $\times 0.5$. The colony formation rate was calculated as the number of colonies/1,500 x100\%.

Animal studies. Female NOD-SCID mice (4 weeks old) were purchased from Beijing HFK Bio-Technology Co. Ltd. (Beijing, China). The studies were approved by the Committee on Ethics of Animal Experiments of Tongji Medical College. The mice were maintained in the accredited animal facility of Tongji Medical
College. Intramuscular tumors were initiated by injecting $1 \times 10^{6}$ tumor cells in $50 \mu \mathrm{l}$ of media into the hind limb of NOD-SCID mice. The sizes of ectopic tumors were monitored by a caliper and calculated by the formula: volume $=(\text { width })^{2} \mathrm{x}$ length $/ 2$. Orthotopic xenograft model of cervical cancer was performed as previously described (20). Briefly, intramuscular tumors were used as donor tumor fragments for orthotopic transplantation into the cervix of NOD-SCID mice. Tumor growth in cervix was dynamically monitored in living mice by optical imaging of luciferase activity using the IVIS Spectrum system (Caliper, Xenogen, USA). After anaesthetization with 3\% pentobarbital sodium, mice were imaged $10 \mathrm{~min}$ after intraperitoneal injection of $100 \mathrm{mg} / \mathrm{kg}$ D-luciferin. Tumor size was measured by three-dimensional reconstruction of image using Living Image version 4.3.1 software.

Statistical analysis. Each experiment was repeated at least three times independently. SPSS (version 13.0) software package was used for statistical analysis. Results are expressed as mean value \pm standard error of the mean (SEM), and interpreted by one-way ANOVA. Differences were considered to be statistically significant at $\mathrm{P}<0.05$. 

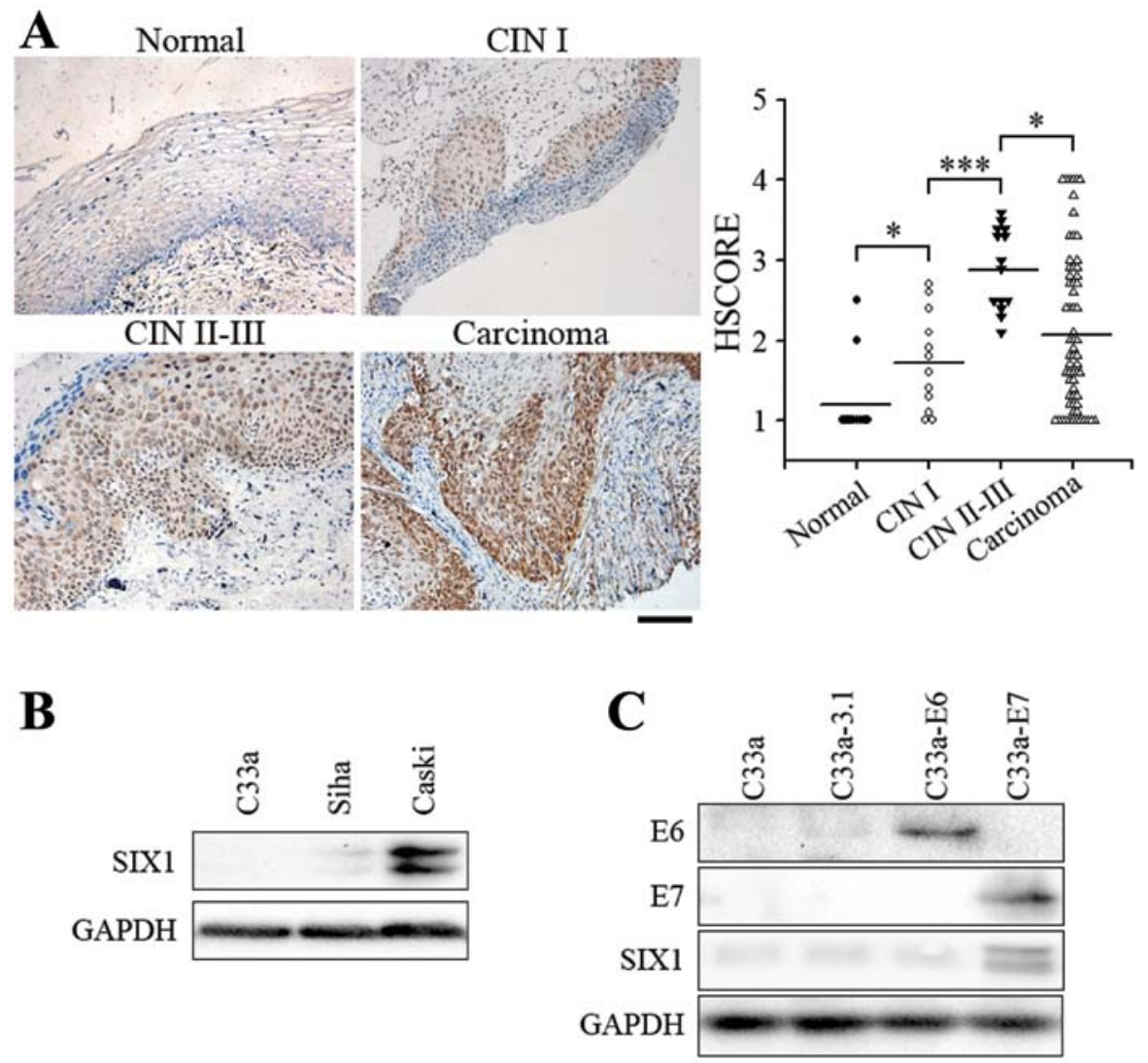

D

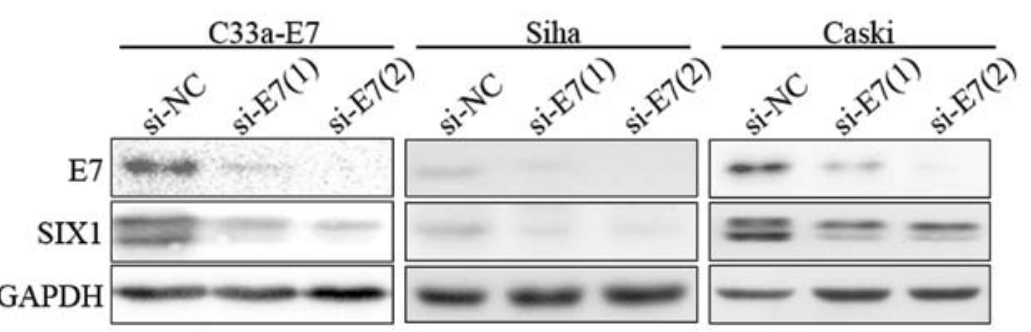

Figure 1. SIX1 expression is increased by HPV oncoprotein in cervical cancer. (A) Immunohistochemical analysis of SIX1 protein in tissue microarray of human specimens. Representative samples of SIX1 staining are shown at x200 magnification (left). Bar, $100 \mu$ m. The immunohistochemical scores (HSCORE) of SIX1 are also shown (right). (B) The expressions of SIX1 in three cervical cancer cell lines were detected by western blot analysis. (C) The expressions of HPV-E6, HPV-E7 and SIX1 in indicated C33a cells were detected by western blot analysis. (D) At 48 h after HPV-E7 siRNA transfection, the expressions of HPV-E7 and SIX1 in cells detected by western blot analysis. ${ }^{*} \mathrm{P}<0.05 ;{ }^{* * *} \mathrm{P}<0.01{ }^{* * * *} \mathrm{P}<0.001$.

\section{Results}

SIX1 expression is increased by HPV oncoprotein in cervical cancer. To determine the role of SIX1 in cervical cancer, we analyzed the expression of SIX1 in each stage of cancer development. SIX1 expression was negligible in normal cervical epithelium, but was significantly increased in low-grade cervical intraepithelial neoplasia (CIN I, precancerous lesion) (Fig. 1A). Higher expression levels of SIX1 were observed in almost all cases of high-grade cervical intraepithelial neoplasia (CIN II-III). SIX1expressing cells shared the same distribution area with the extent of intraepithelial neoplasia (Fig. 1A). In three cervical squamous cell carcinoma cell lines, the expression of SIX1 was very low in HPV-negative C33a cells, slightly elevated in Siha cells which contain 1-2 copies of HPV DNA, and much higher in Caski cells which contain
60-600 copies of HPV DNA (Fig. 1B), suggesting that HPV infection increased the expression of SIX1. We then further tested whether SIX1 was induced by E6 or E7 oncoprotein, the driving force of cervical cancer. Overexpression of E7, but not E6, in HPV-negative C33a cells induced SIX1 expression (Fig. 1C). Consistently, when E7 were knocked down in C33a-E7, Siha or Caski cells, the expression of SIX1 was significantly decreased (Fig. 1D). These results suggested that HPV-E7 is one of the factors that are required for inducing the expression of SIX1. Moreover, the correlation of SIX1 expression to the progression of cervical cancer suggested that SIX1 might play an important role in tumorigenesis and progression of cervical cancer.

SIX1 may modulate the expression of multiple genes to promote tumor cell proliferation. To identify the effect of SIX1 on the development of cervical cancer, bioinformatic 
A

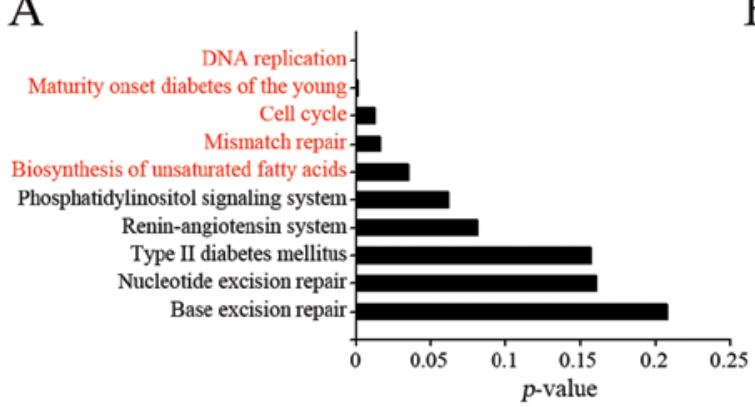

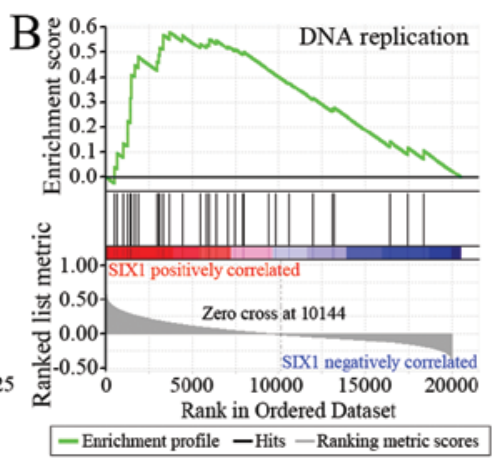

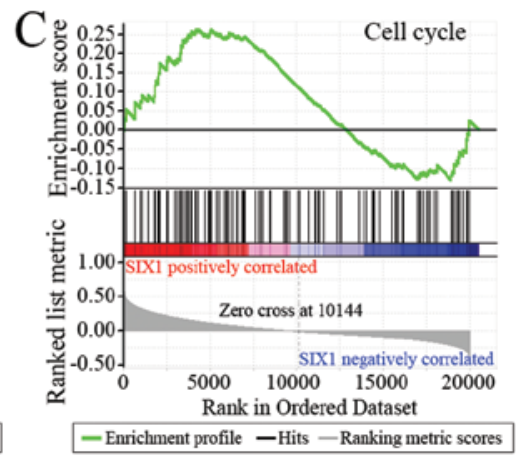

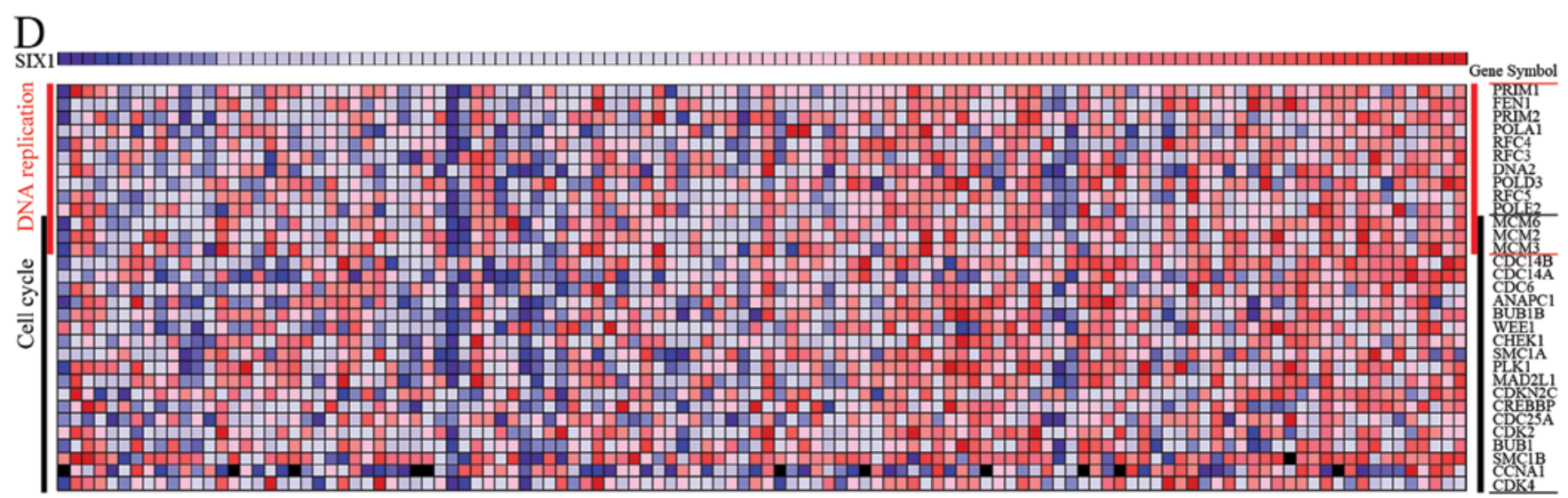

Figure 2. SIX1 may modulate the expression of multiple genes to promote tumor cell proliferation. (A) Pathway analysis of the genes significantly upregulated in SIX1-high expression group was performed using Onto-Tools. (B and C) Enrichment plot for the gene sets of (B) DNA replication pathway and (C) cell cycle pathway by GSEA. Bottom, plot of the ranked list of all genes. Y-axis, value of the ranking metric; $x$-axis, the rank for all genes. Middle, the location of genes from indicated pathway within the ranked list. Top, the running enrichment score (green line) for the gene set as the analysis walks along the ranked list. (D) Heat map. The expression profile of the genes related to DNA replication and the cell cycle, based on the identification of differential expression by both edgeR-Onto and GSEA package. Columns represent different samples and are plotted according to SIX1 expression increased from left to right.

analysis was performed to predict the effect of SIX1 on gene expression in cervical cancer. The RNA-sequencing data of 116 cervical cancer specimens was obtained from TCGA database. The specimens were divided into SIX1-low expression group $(\mathrm{n}=58)$ and SIX1-high expression group $(\mathrm{n}=58)$. Bioconductor edge $\mathrm{R}$ package was used to identify the genes which were significantly up-regulated in SIX1-high expression group. Subsequent pathway analysis indicated five pathways which were significantly enriched in the genes potentially upregulated by SIX1 (Fig. 2A). Two of the pathways were correlated with cell proliferation: DNA replication and the cell cycle. Further analysis by using GSEA package confirmed that the enrichment of the upregulated genes related to DNA replication was significantly correlated with the increase of SIXI gene expression (Fig. $2 \mathrm{~B}, \mathrm{P}<0.05$, FDR $<0.25$ ). The enrichment of the upregulated genes related to cell cycle was correlated, although without statistical significance, with the increase of SIXI gene expression (Fig. 2C). Importantly, the analysis with both edgeR-Onto and GSEA methods showed that the upregulated genes included many genes related to DNA replication and the cell cycle, and that these genes could be considered as candidate genes which might be up-regulated by SIX1 (Fig. 2D). These results implied that SIX1 may regulate the expression of the genes involved in DNA replication to promote tumor cell proliferation.

SIX1 regulates the expression of genes responsible for DNA replication and enhances DNA synthesis. Based on the above results, we overexpressed SIX1 in C33a cells (Fig. 3A) to investigate the regulatory effect of SIX1 on DNA replication genes (Fig. 3B). SIX1 expression was knocked down in Caski cells (Fig. 3C) to further confirm the effect of SIX1 (Fig. 3D). The results showed that SIX1 could upregulate the expression of several genes coding for the proteins in five complexes related to DNA replication, including MCM complex (MCM2, MCM3, MCM6),DNA polymerase $\alpha$-primase complex (POLA1,PRIM1, PRIM2), clamp loader (RFC3, RFC4, RFC5), DNA polymerase $\delta$ complex (POLD3) and DNA polymerase $\varepsilon$ complex (POLE2). These five complexes play critical roles in three important steps of DNA replication initiation: DNA unwinding, synthesis of primer, and switching from DNA polymerase $\alpha$ to polymerase $\delta$ or polymerase $\varepsilon$ (Fig. 4A). Consistently, BrdU incorporation assay indicated that DNA synthesis was more active in SIX1 highly expressed cervical cancer cells (Fig. 4B).

SIX1 modulates the cell cycle and promotes G1 to S phase progression. In accordance with the potential of SIX1 to 

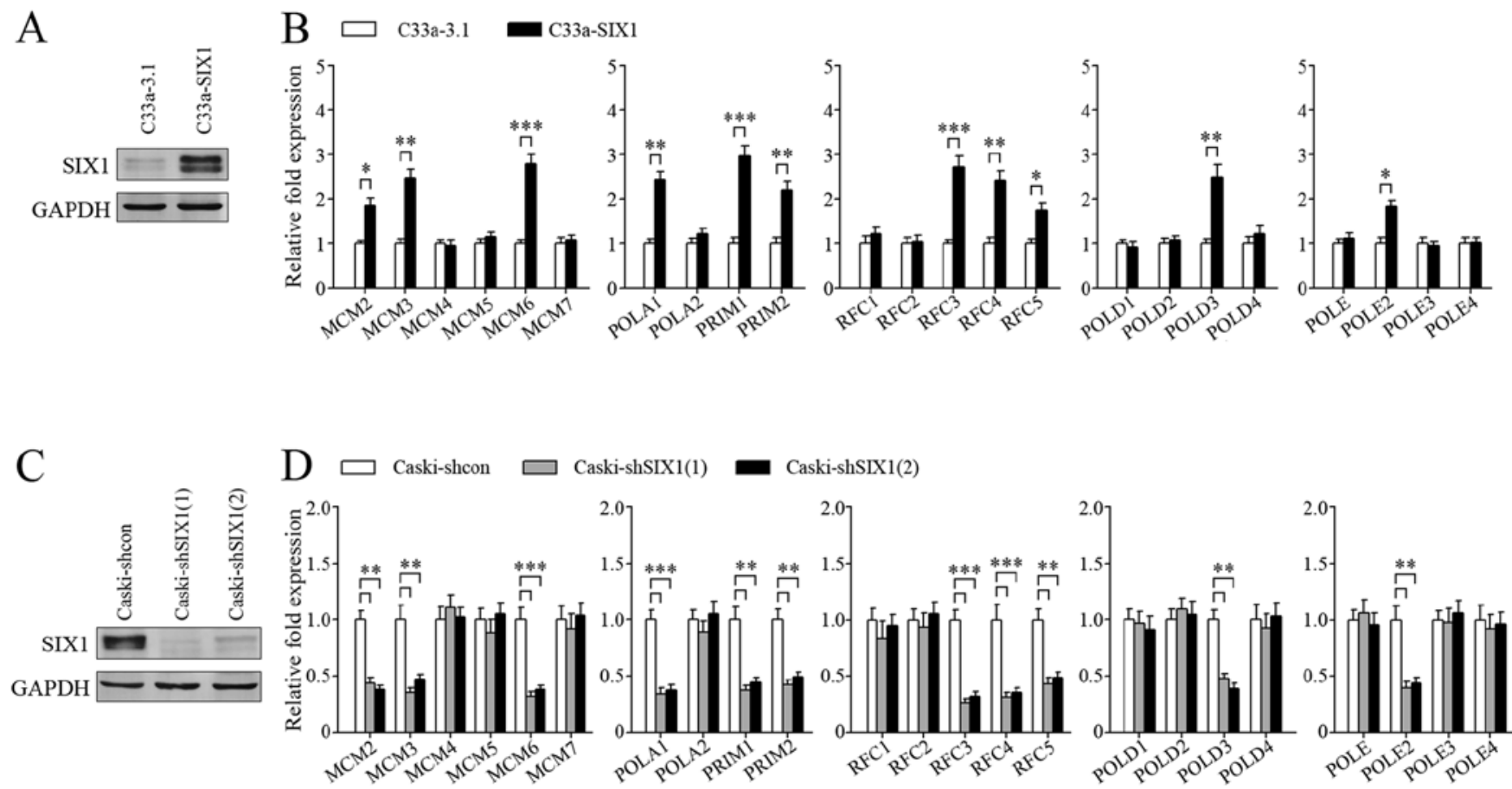

Figure 3. SIX1 regulates the expression of genes responsible for DNA replication. (A and B) In C33a-3.1 and C33a-SIX1 cells, SIX1 expression was detected by (A) western blot analysis, and the expression levels of indicated genes were detected by (B) real-time RT-PCR. (C and D) Two independent shRNAs were used to knockdown SIX1 expression in Caski cells. SIX1 expression was detected by (C) western blot analysis and the expression levels of indicated genes were detected by (D) real-time RT-PCR. ${ }^{*} \mathrm{P}<0.05 ;{ }^{* *} \mathrm{P}<0.01 ;{ }^{* * *} \mathrm{P}<0.001$.

\begin{tabular}{|c|c|c|c|c|c|}
\hline \multirow{3}{*}{$\begin{array}{l}\mathrm{A} \text { DNA } \\
\text { unwinding }\end{array}$} & \multicolumn{3}{|c|}{ MCM complex (helicase) } & & \\
\hline & MCM2 & MCM3 & MCM4 & & \\
\hline & MCM5 & MCM6 & MCM7 & & \\
\hline \multirow{2}{*}{ priming } & \multicolumn{4}{|c|}{ DNA polymerase $\alpha$-primase complex } & \\
\hline & $\alpha 1$ & $\alpha 2$ & PRIM1 & PRIM2 & \\
\hline \multirow{6}{*}{ 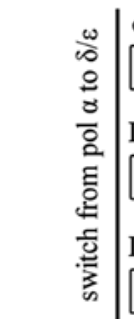 } & \multicolumn{5}{|c|}{ clamp loader } \\
\hline & RFC1 & RFC2 & RFC3 & RFC4 & RFC5 \\
\hline & \multicolumn{4}{|c|}{ DNA polymerase $\delta$ complex } & \\
\hline & $\delta 1$ & $\delta 2$ & $\delta 3$ & $\delta 4$ & \\
\hline & \multicolumn{4}{|c|}{ DNA polymerase $\varepsilon$ complex } & \\
\hline & \begin{tabular}{|l|}
$\varepsilon 1$ \\
\end{tabular} & $\varepsilon 2$ & $\varepsilon 3$ & $\varepsilon 4$ & \\
\hline
\end{tabular}

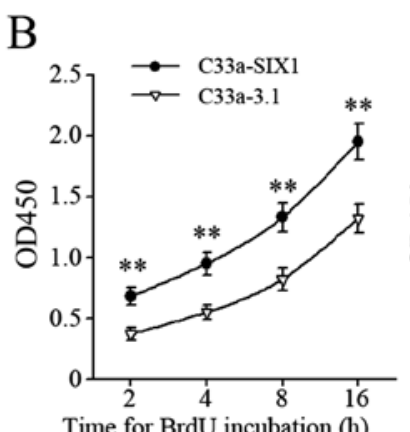

Figure 4. SIX1 regulates the genes coding for the proteins in five protein complexes and enhances DNA synthesis. (A) The genes that are upregulated by SIX1 are highlighted in gray boxes of the corresponding complexes. (B) Tumor cells were incubated with BrdU solution for indicated time. DNA synthesis was measured by BrdU incorporation. ${ }^{*} \mathrm{P}<0.05 ;{ }^{* *} \mathrm{P}<0.01 ;{ }^{* * * *} \mathrm{P}<0.001$. promote DNA replication, overexpression of SIX1 in C33a cells resulted in a decrease of the percentage of cells in G0/G1 phase and a concomitant increase of the percentage of cells in S phase (Fig. 5A), indicating more active cell proliferation. After synchronization by serum starvation and following serum addition, C33a-SIX1 cells progressed into $\mathrm{S}$ phase faster than corresponding control cells (Fig. 5B), and knockdown of SIX1 in Caski cells resulted in the opposite effect (Fig. 5C and D). These results demonstrated that SIX1 modulated the cell cycle and promoted G1 to S phase progression.

SIXI promotes tumor cell proliferation in vitro. We next investigated whether SIX1 could promote tumor cell proliferation. Overexpression of SIX1 in C33a cells significantly, although slightly, promoted anchorage-dependent cell growth, while knockdown of SIX1 in Caski cells decreased anchorage-dependent cell growth (Fig. 6A). Intriguingly, in colony-forming assays in soft agar, the tumor cells with highly expressed SIX1 showed remarkably larger size colonies (Fig. 6B) and much higher colony formation rate (Fig. 6C), indicating that SIX1 could more efficiently promote anchorage-independent cell growth.

SIX1 promotes tumor growth in vivo. Expression of the proliferation marker Ki-67, was significantly higher in SIX1 highly expressed cervical cancer specimens (Fig. 7A). Comparing with corresponding control groups, the percentage of Ki67-positive tumor cells was increased in C33a-SIX1 tumors and decreased in Caski-shSIX1 tumors (Fig. 7B), indicating more active cell proliferation in SIX1 highly expressed tumors. In line 
A
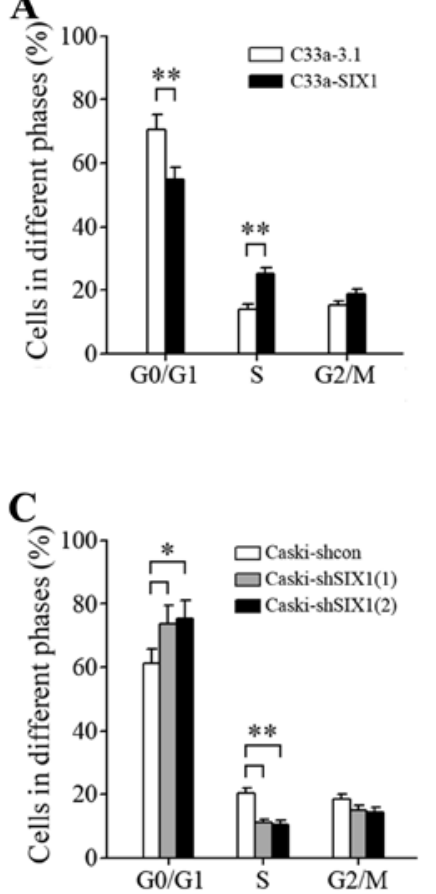

B

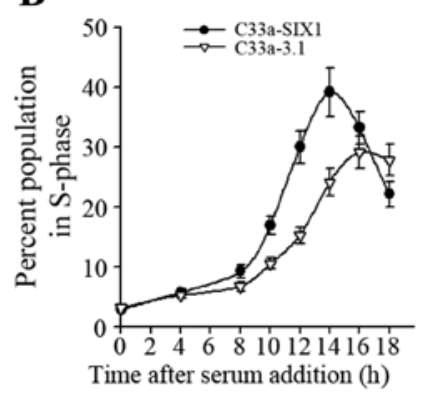

D

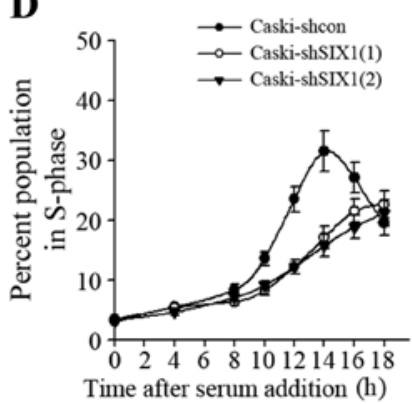

Figure 5. SIX1 modulates the cell cycle and promotes G1-to-S phase progression. (A and C) The cells in different phases of the cell cycle were quantified by flow cytometry. (B and D) Progression into S phase following serum addition to the cultures after serum starvation. At each time point after serum addition, the population of cells in $\mathrm{S}$ phase was determined by flow cytometric analysis. ${ }^{*} \mathrm{P}<0.05 ;{ }^{* *} \mathrm{P}<0.01 ;{ }^{* * *} \mathrm{P}<0.001$.

with this, in both ectopic (Fig. 7C) and orthotopic (Fig. 7D) xenograft models of cervical cancer, overexpression of SIX1 in C33a cells significantly promoted tumor growth, while knockdown of SIX1 in Caski cells decreased tumor growth. Taken together, these results demonstrated that SIX1 could promote not only tumor cell proliferation in vitro, but also tumor growth in vivo.

\section{Discussion}

Malignant proliferation is the fundamental trait of tumor cells. The initiation of DNA replication represents a key process for cell proliferation, and has a marked impact on tumorigenesis and progression $(19,21)$. The factors that promote DNA replication are organized in multiprotein complexes that coordinately help to recognize regions of origin, unwind DNA and promote replisome formation (21). The data in this study showed that SIX1 could modulate the expression of several genes related to DNA replication, thus accelerating G1 to $S$ phase progression, promoting the proliferation of cervical cancer cells in vitro, and promoting the growth of cervical cancer in vivo.

The expression of SIX1 was closely correlated with the progression of cervical cancer as shown by our data. High levels of SIX1 expression were observed in almost all regions that developed intraepithelial neoplasia, suggesting that SIX1 might play an important role in tumorigenesis and progression of cervical cancer. Our data showed that the expression of SIX1 was positively correlated with the copy number of HPV DNA in cervical squamous cell carcinoma cells. These results
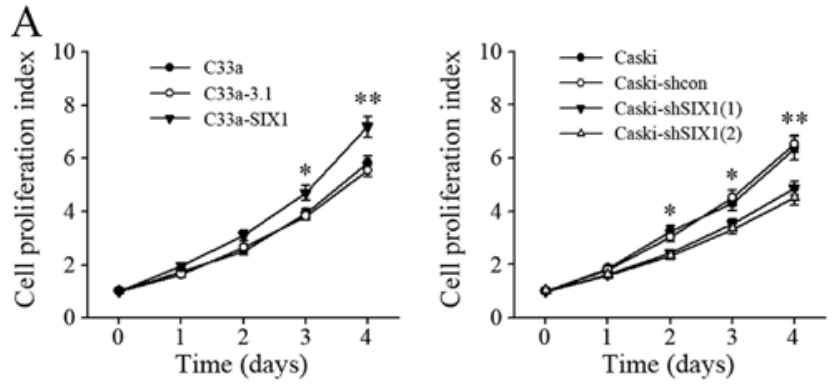
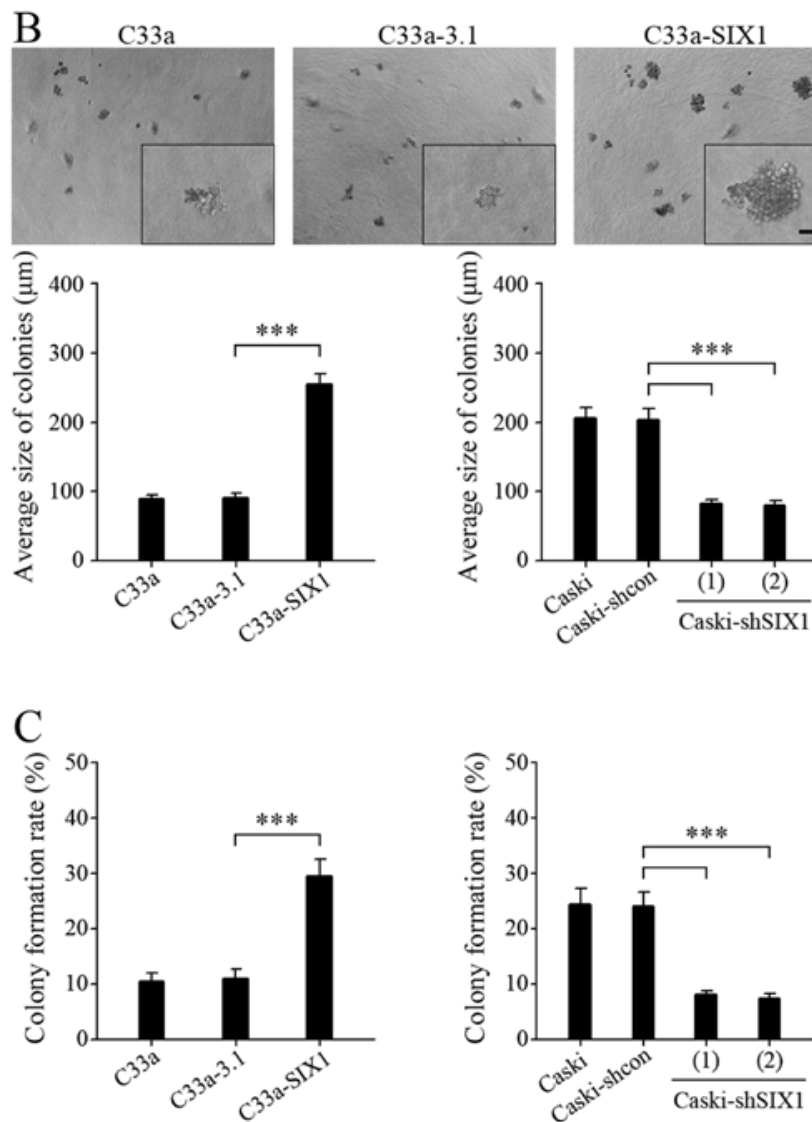

Figure 6. SIX1 promotes tumor cell proliferation in vitro. (A) The proliferation index of indicated cells was determined as described in Materials and methods. (B and C) Soft agar colony formation assay. The representative images are shown at $\times 40$ and $\times 200$ (inserts) magnification (B, upper panels). Bar, $100 \mu \mathrm{m}$. The (B, lower panels) average size of colonies and $(\mathrm{C})$ colony formation rate were calculated. ${ }^{*} \mathrm{P}<0.05 ;{ }^{* * *} \mathrm{P}<0.01 ;{ }^{* * *} \mathrm{P}<0.001$.

were also supported by a report that SIX1 was significantly increased in HPV-immortalized normal human keratinocytes (4). Moreover, we demonstrated that SIX1 expression in cervical cancer cells could be modulated by HPV oncoprotein. HPV-E7, but not E6, was required for sustaining the expression of SIX1 gene in cervical cancer cells, including C33a-E7, Siha and Caski cells. These results also implied that HPV-E7 oncoprotein might continuously promote the progression of cervical cancer after the formation of tumor by upregulating the expression of SIX1.

The general process of DNA replication initiation involves several key steps: loading of helicase at replication origins, unwinding of DNA by helicase, the synthesis of RNA primer 

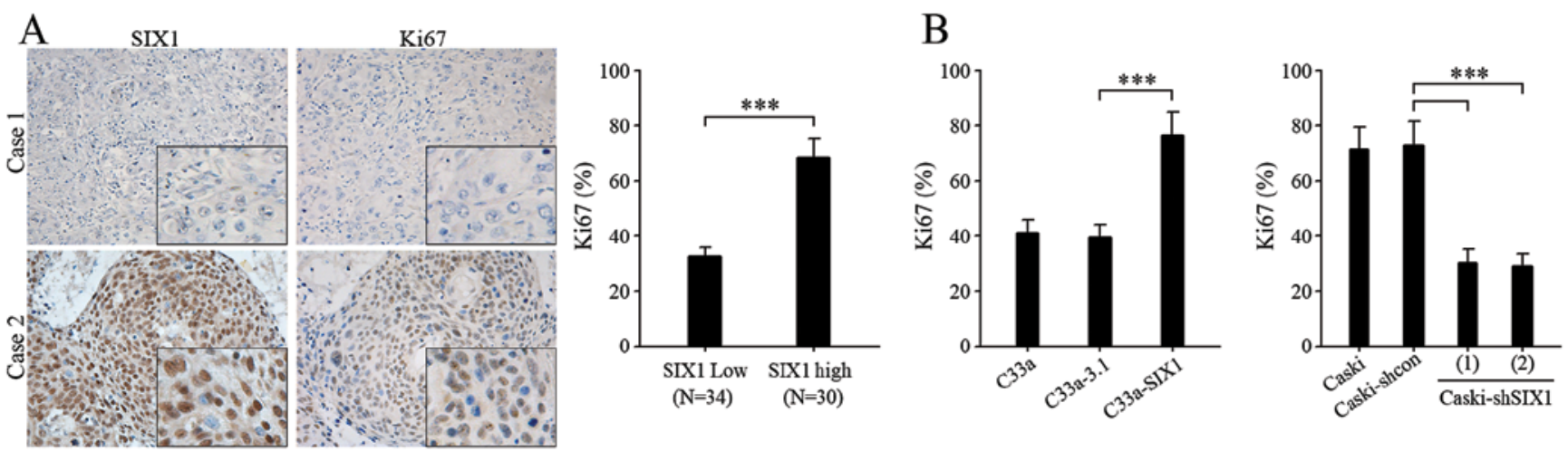

$\mathrm{C}$

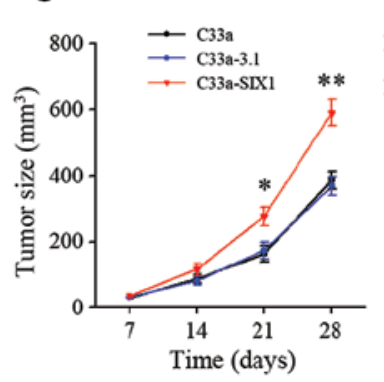

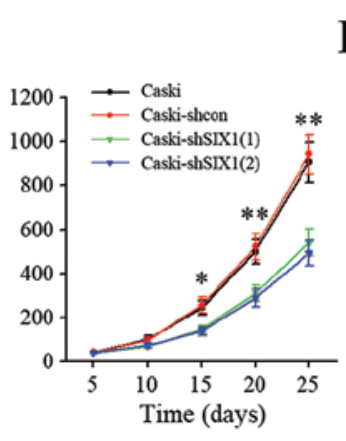

$\mathrm{D}$

Figure 7. SIX1 promotes tumor growth in vivo. (A) Immunohistochemical analysis of SIX1 and Ki67 proteins in tissue microarrays of human specimens Representative patterns of staining are shown at $x 400$ magnification (left). HSCORE $\leq 2.0$ was classified as low-SIX1 expression, and HSCORE >2.0 was classified as high-SIX1 protein expression. The percentage of Ki67-positive tumor cells was calculated as described in Materials and methods (right). (B) Immunohistochemical analysis of Ki67 protein in tumors formed by the indicated cells. The percentages of Ki67-positive tumor cells were calculated. A total of 10 ectopic tumors and 10 orthotopic tumors per group were analyzed. (C) After inoculating the indicated cells into the hind limb of NOD-SCID mice, the size of tumor was closely monitored by caliper. Tumor size was calculated (n=10 per group). (D) After orthotopic transplantation of tumors pre-formed by the indicated cells, the size of tumor was closely monitored by three-dimensional reconstruction of in vivo bioluminescence images (left). Tumor size was calculated ( $\mathrm{n}=10$ per group, right). ${ }^{*} \mathrm{P}<0.05 ;{ }^{* *} \mathrm{P}<0.01 ;{ }^{* * * *} \mathrm{P}<0.001$.

by primase, elongation of primer by DNA polymerase $\alpha$, and then the replacement of DNA polymerase $\alpha$ with polymerase $\delta$ or polymerase $\varepsilon$ for extending DNA strands $(22,23)$. SIX1 could modulate the expression of the genes that mainly affect the initiation of DNA replication as shown by out data. The upregulation of multiple genes by SIX1 might increase the efficiency of DNA unwinding, synthesis of primer, and switching from DNA polymerase $\alpha$ to polymerase $\delta$ or polymerase $\varepsilon$.

MCM complex serves as the primary helicase for unwinding DNA during replication (21). Members of MCM family are proposed as diagnostic or prognostic markers in various cancers due to their increased proliferative potential (24). Studies have demonstrated that CDC6 is necessary for the loading of MCM complex at replication origins (25). Our data in this study showed that SIX1 upregulated the expression of MCM2, MCM3 and MCM6. Bioinformatic analysis also suggested that SIX1 could upregulate the expression of CDC6. Therefore, SIX1 might accelerate the initiation of DNA replication by upregulating the expression of these genes.

The synthesis of RNA primer by primase and the following elongation of the primer by DNA polymerase $\alpha$ are required for DNA replication in eukaryotes (23). The primase consists of two subunits, p49 (PRIM1) and p58 (PRIM2), both of which were upregulated by SIX1. Moreover, SIX1 could upregulate the expression of polymerase $\alpha 1$ (POLA1). The increased expression of these genes might make the initiation of DNA replication more efficient by improving the efficiency of primer synthesis. After the synthesis and elongation of primer, the synthesis of new DNA strands was catalyzed by DNA polymerase $\delta$ and polymerase $\varepsilon$. The switch from polymerase $\alpha$ to polymerase $\delta$ and polymerase $\varepsilon$ is mediated by replication factor C (RFC) $(26,27)$. DNA polymerases and RFC are important not only for DNA replication, but also for cell cycle control (26). RFC3 and RFC4 were reported to promote tumor cell proliferation $(28,29)$. High RFC3 expression was associated with poor prognosis in a variety of cancers (28). Importantly, SIX1 not only increased the expression of DNA polymerase $\delta 3$ (POLD3) and polymerase $\varepsilon 2$ (POLE2), but also upregulated the expression of RFC (RFC3, RFC4, RFC5). Therefore, SIX1 might not only promote the initiation of DNA replication by increasing the expression of primase and DNA polymerase $\alpha$, but also promote the further synthesis of DNA strands by upregulating the expression of DNA polymerase $\delta 3$, polymerase $\varepsilon 2$ and RFC.

SIX1 could significantly promote tumor cell proliferation in vitro. Correspondingly, ectopic and orthotopic xenograft models of cervical cancer further demonstrated that SIX1 could promote tumor growth in vivo. Intriguingly, colony formation assays in soft agar showed that SIX1 remarkably promoted anchorage-independent cell growth, suggesting that the promoting effect of SIX1 on tumor cell proliferation might promote not only the development of primary tumor but also the proliferation of disseminated tumor at metastatic sites. 
In summary, this study revealed the important role of SIX1 in modulating DNA replication. SIX1 upregulated the expression of multiple key genes involved in the initiation of DNA replication. These genes coordinate to accelerate G1 to S phase progression, promote proliferation of cervical cancer cells and the growth of cervical cancer. These results implied that SIX1 might play a crucial role in the tumorigenesis and progression of cervical cancer, and also suggested that targeting SIX1 might have significant therapeutic value in cervical cancer treatment.

\section{Acknowledgements}

We thank Dr Qi-Lin Ao and Dr Shuang Guo for reviewing the histology data. This study was supported by National Science Foundation of China (nos. 81072135, 81372801, 81172466, 81072132 and 81372781$)$.

\section{References}

1. Moody CA and Laimins LA: Human papillomavirus oncoproteins: pathways to transformation. Nat Rev Cancer 10: 550-560, 2010.

2. Ferenczy A and Franco E: Persistent human papillomavirus infection and cervical neoplasia. Lancet Oncol 3: 11-16, 2002.

3. Schiffman M, Castle PE, Jeronimo J, Rodriguez AC and Wacholder S: Human papillomavirus and cervical cancer. Lancet 370: 890-907, 2007.

4. Wan F, Miao X, Quraishi I, Kennedy V, Creek KE and Pirisi L: Gene expression changes during HPV-mediated carcinogenesis: a comparison between an in vitro cell model and cervical cancer. Int J Cancer 123: 32-40, 2008.

5. McCoy EL, Iwanaga R, Jedlicka P, et al: Six1 expands the mouse mammary epithelial stem/progenitor cell pool and induces mammary tumors that undergo epithelial-mesenchymal transition. J Clin Invest 119: 2663-2677, 2009.

6. Christensen KL, Brennan JD, Aldridge CS and Ford HL: Cell cycle regulation of the human Six1 homeoprotein is mediated by APC(Cdh1). Oncogene 26: 3406-3414, 2007.

7. Micalizzi DS, Wang CA, Farabaugh SM, Schiemann WP and Ford HL: Homeoprotein Six 1 increases TGF-beta type I receptor and converts TGF-beta signaling from suppressive to supportive for tumor growth. Cancer Res 70: 10371-10380, 2010.

8. Ford HL, Kabingu EN, Bump EA, Mutter GL and Pardee AB: Abrogation of the G2 cell cycle checkpoint associated with overexpression of HSIX1: a possible mechanism of breast carcinogenesis. Proc Natl Acad Sci USA 95: 12608-12613, 1998.

9. Coletta RD, Christensen K, Reichenberger KJ, et al: The Six 1 homeoprotein stimulates tumorigenesis by reactivation of cyclin A1. Proc Natl Acad Sci USA 101: 6478-6483, 2004.

10. Cho YS, Kang JW, Cho M, et al: Down modulation of IL-18 expression by human papillomavirus type 16 E6 oncogene via binding to IL-18. FEBS Lett 501: 139-145, 2001.

11. Sun C, Li N, Yang Z, et al: miR-9 Regulation of BRCA1 and ovarian cancer sensitivity to cisplatin and PARP inhibition. J Natl Cancer Inst 105: 1750-1758, 2013.
12. Xiong T, Zhao Y, Hu D, et al: Administration of calcitonin promotes blastocyst implantation in mice by up-regulating integrin beta3 expression in endometrial epithelial cells. Hum Reprod 27: 3540-3551, 2012.

13. Shen Y, Li Y, Ye F, Wang F, Lu W and Xie X: Identification of suitable reference genes for measurement of gene expression in human cervical tissues. Anal Biochem 405: 224-229, 2010.

14. Wang W, Fang Y, Sima N, et al: Triggering of death receptor apoptotic signaling by human papillomavirus 16 E2 protein in cervical cancer cell lines is mediated by interaction with c-FLIP. Apoptosis 16: 55-66, 2011.

15. Robinson MD, McCarthy DJ and Smyth GK: edgeR: a Bioconductor package for differential expression analysis of digital gene expression data. Bioinformatics 26: 139-140, 2010.

16. Anders S, McCarthy DJ, Chen Y, et al: Count-based differential expression analysis of RNA sequencing data using R and bioconductor. Nat Protoc 8: 1765-1786, 2013.

17. Draghici S, Khatri P, Bhavsar P, Shah A, Krawetz SA and Tainsky MA: Onto-Tools, the toolkit of the modern biologist: Onto-Express, Onto-Compare, Onto-Design and Onto-Translate. Nucleic Acids Res 31: 3775-3781, 2003.

18. Subramanian A, Tamayo P, Mootha VK, et al: Gene set enrichment analysis: a knowledge-based approach for interpreting genome-wide expression profiles. Proc Natl Acad Sci USA 102: 15545-15550, 2005.

19. Mazurek A, Luo W, Krasnitz A, Hicks J, Powers RS and Stillman B: DDX5 regulates DNA replication and is required for cell proliferation in a subset of breast cancer cells. Cancer Discov 2: 812-825, 2012.

20. Cairns RA and Hill RP: A fluorescent orthotopic model of metastatic cervical carcinoma. Clin Exp Metastasis 21: 275-281, 2004.

21. Costa A, Hood IV and Berger JM: Mechanisms for initiating cellular DNA replication. Annu Rev Biochem 82: 25-54, 2013.

22. Soultanas P: Loading mechanisms of ring helicases at replication origins. Mol Microbiol 84: 6-16, 2012.

23. Urban M, Joubert N, Purse BW, Hocek M and Kuchta RD: Mechanisms by which human DNA primase chooses to polymerize a nucleoside triphosphate. Biochemistry 49: 727-735, 2010.

24. Gan N, Du Y, Zhang W and Zhou J: Increase of Mcm3 and $\mathrm{Mcm} 4$ expression in cervical squamous cell carcinomas. Eur J Gynaecol Oncol 31: 291-294, 2010.

25. Cook JG, Park CH, Burke TW, et al: Analysis of Cdc6 function in the assembly of mammalian prereplication complexes. Proc Natl Acad Sci USA 99: 1347-1352, 2002.

26. Masuda Y, Suzuki M, Piao J, Gu Y, Tsurimoto T and Kamiya K: Dynamics of human replication factors in the elongation phase of DNA replication. Nucleic Acids Res 35: 6904-6916, 2007.

27. Maga G, Stucki M, Spadari S and Hubscher U: DNA polymerase switching: I. Replication factor C displaces DNA polymerase alpha prior to PCNA loading. J Mol Biol 295: 791-801, 2000.

28. Lockwood WW, Thu KL, Lin L, et al: Integrative genomics identified RFC3 as an amplified candidate oncogene in esophageal adenocarcinoma. Clin Cancer Res 18: 1936-1946, 2012.

29. Arai M, Kondoh N, Imazeki N, et al: The knockdown of endogenous replication factor $\mathrm{C} 4$ decreases the growth and enhances the chemosensitivity of hepatocellular carcinoma cells. Liver Int 29: 55-62, 2009. 